\title{
MENINGIOMAS SUPRATENTORIAIS
}

\section{DIAGNÓSTICO, RESULTADOS CIRÚRGICOS E COMPLICAÇÕES}

\author{
EBERVAL GADELHA FIGUEIREDO, PAULO HENRIQUE AGUIAR, \\ BELARMINO FONSECA CÓRDOBA, YASSUNORI FUJIMOTO, \\ JOSÉ MARCUS ROTTA, RAUL MARINO JR.
}

\begin{abstract}
RESUMO - Os meningiomas são tumores benignos originados de células da aracnóide e representam os tumores intracranianos assintomáticos mais comuns. Estudamos 69 casos de meningiomas supratentoriais operados pelo Grupo de Tumores da Disciplina de Neurocirurgia do Hospital das Clínicas da Faculdade de Medicina da Universidade de São Paulo no período de setembro-1995 a setembro-1997. Foram analisados idade, sexo, grau de edema, localização, complicações cirúrgicas e mortalidade. O grau de edema foi definido pelos exames de imagem (TC e RNM). A média de idade foi 58 anos; 47 pacientes eram do sexo feminino. Houve discreta predominância $(38,7 \%)$ de pacientes com edema grau II. Vinte e nove tumores eram parassagitais e 40 de convexidade. Obteve-se grau de ressecção Simpson I em 48 casos, II em 18 e III em dois (casos com implante em seio sagital pérvio). Complicações ocorreram em 9 casos (déficits transitórios, $n=6$; déficit permanente, $n=1$; e infecções, $n=2$ ). Óbito ocorreu em dois pacientes relacionados à idade avançada e a tumores parassagitais, decorrendo de complicações vasculares. A morbi-mortalidade esteve relacionada à idade, a tumores falcinos e à tentativa de ressecção radical de tumores implantados em seios venosos pérvios. Não houve correlação entre a morbi-mortaliade e o grau de edema.
\end{abstract}

PALAVRAS-CHAVE: meningiomas supratentoriais, cirurgia, edema cerebral, seios venosos.

\section{Supratentorial meningiomas: clinical evaluation, surgical results and complications.}

ABSTRACT - Meningiomas are benign tumors arisising from arachnoid cells and represent the commonest asymptomatic intracranial tumors. We analysed 69 supratentorial meningiomas managed by the Neurosurgical Tumor Group of the Clinics Hospital of Medicine School of São Paulo University (September 1995 to September 1997). Age, sex, edema degree, tumor site, surgical complications and mortality were studied. Edema degree was defined by radiological methods (CT and MRI). Forty-seven patients were women and average age was 58 years. Type II of edema degree was predominant (38.7\%). Twenty-nine patients had parasagital meningiomas and 40 presented convexity tumors. Simpson I resection was obtained in 48 procedures, II in 18 and III in two surgical removals. Nine cases complicated (transitory deficits, 6; permanent deficit, 1; and infection, 2). Death occurred in two patients. Morbity and mortality had relation with age, falx tumors and attempt of radical surgical removal. Edema degree did not modify mortality and morbity rates.

KEY WORDS: supratentorial meninigiomas, surgery, brain edema, venous sinus.

Os meningiomas são tumores originados de células das vilosidades aracnóideas e constituem os tumores intracranianos assintomáticos mais comuns. Representam cerca de 13-18\% dos tumores intracranianos primitivos ${ }^{1,2}$, atingindo mais frequentemente indivíduos com idade entre 20 e 60 anos e acomentendo principalmente o sexo feminino. ${ }^{3}$

Disciplina de Neurocirurgia do Hospital das Clínicas (HC) da Faculdade de Medicina da Universidade de São Paulo (FMUSP). Aceite: 8-abril-1998.

Dr. Paulo Henrique Aguiar - Rua Maestro Torquato Amore 332, Apto 12, Bloco I - 05622-050 São Paulo SP - Brasil. 
Apresentamos uma série de 69 casos de meningiomas supratentoriais consecutivamente operados, analisando aspectos clínicos, radiológicos - tomografia computadorizada (TC) de crânio, ressonância nuclear magnética (RNM), angiografia cerebral e angioressonância - e resultados cirúrgicos e evolução.

\section{MATERIAL E MÉTODOS}

Foram estudados 69 pacientes com diagnóstico de meningioma que foram consecutivamente operados no Grupo de Tumores do Serviço de Neurocirurgia do HC-FMUSP no período de setembro-1995 a setembro1997. Dados de idade, sexo, grau de edema, localização, grau de ressecção, complicações cirúrgicas e mortalidade foram analisados.

Todos os pacientes com meningiomas parassagitais foram submetidos a angiografia ou angioressonância para determinar a relação do tumor com o seio sagital superior, assim como sua patência, objetivando melhor planejamento cirúrgico. O grau de edema foi definido pela TC e/ou RNM em todos os casos e classificado como: grau zero quando limitado a não mais que $1,0 \mathrm{~cm}$ da lesão; grau I em casos em que se estendia no máximo até a metade de um hemisfério (Fig 1), e grau II quando acometia mais da metade de um hemisfério4. O grau de ressecção foi classificado segundo o sistema de gradação de Simpson5.

Todos os pacientes receberam medicação anticonvulsivante, independentemente de história prévia de crises convulsivas. Nos casos classificados como edema grau II ou naqueles com outra gradação mas com déficit motor ou de linguagem, instituiu-se dexametasona na dose de $16 \mathrm{mg} / \mathrm{dia}$, pelo menos nos cinco dias prévios à cirurgia. Manitol $20 \%$ foi utilizado no intra-operatório em casos de edema refratário ao corticóide. Após a cirurgia os pacientes foram encaminhados à unidade de terapia intensiva. TC de crânio foi realizada nas primeiras 24 horas de pós-operatório para avaliar o grau de ressecção da lesão e a eventual presença de hematomas (Fig 2). O corticóide foi continuado no período pós-operatório de pacientes com edema graus I e II e retirado ambulatorialmente.

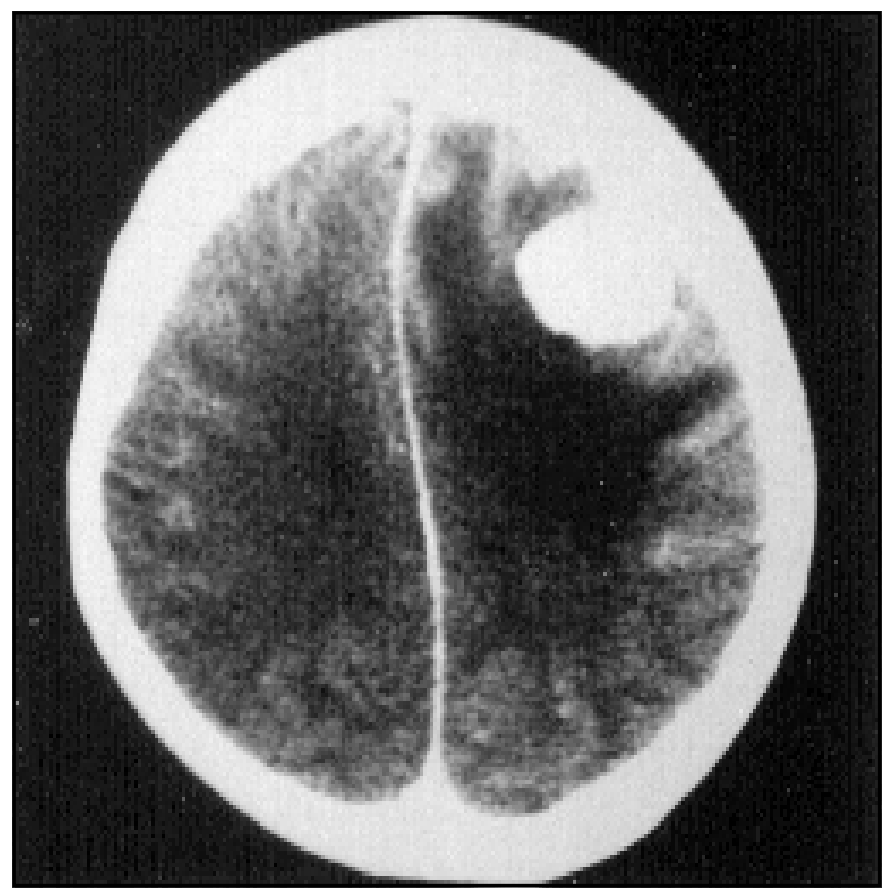

Fig 1. Tomografia axial de crânio após injeção de contraste iodado mostrando meningioma de convexidade frontal e importante edema perilesional que acomete principalmente a substância branca e provoca desvio da linha mediana. 


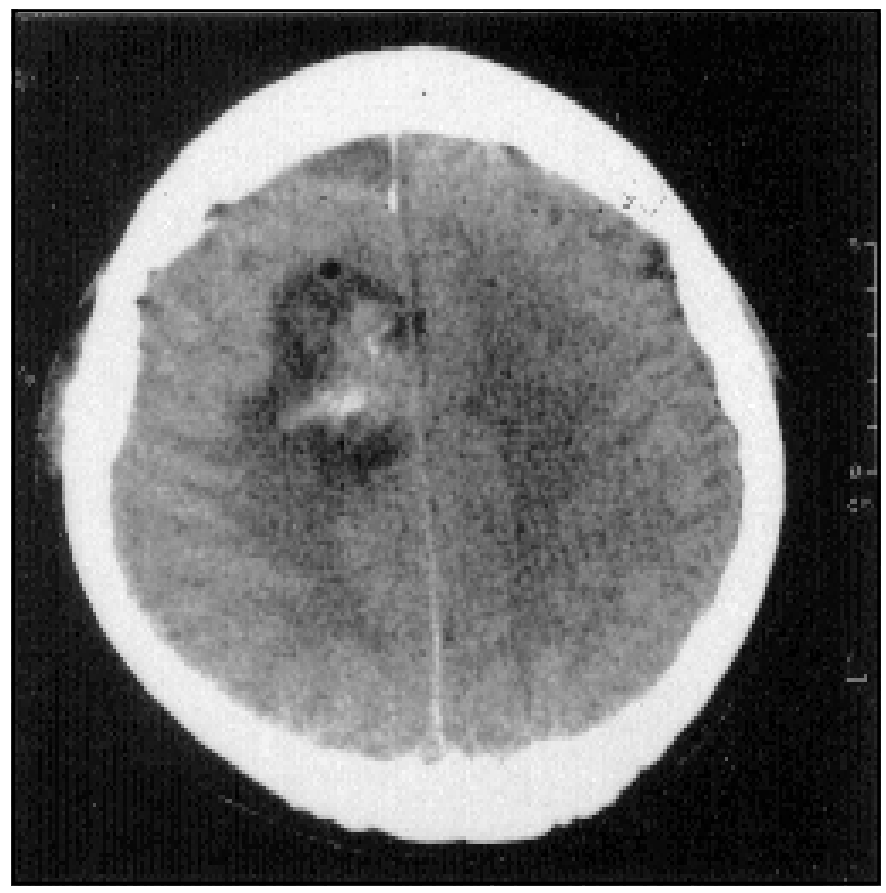

Fig 2. Tomografia axial de crânio após injeção de contraste iodado realizada no primeiro dia pós-operatório de um meningioma falcino revelando ressecção total da lesão. O aspecto pré-operatório pode ser visto na Fig 3.

\section{RESULTADOS}

Quarenta e sete pacientes eram do sexo feminino e 22 eram do sexo masculino. A média de idade foi 58 anos (mínima 20 e máxima 82 anos). 30,9\% dos pacientes apresentavam edema grau zero, 30,4\% grau I e 38,7\% grau II. Meningiomas parassagitais totalizaram 29 casos (14 falcinos) (Fig 3); 28 eram de convexidade frontal e parietal; e 12 eram de convexidade temporal (2 de terço externo de asa de osso esfenóide).

O grau de ressecção segundo o sistema de gradação de Simpson foi: I em 48 casos (69,5\%); II em 18 (26\%); e III em 3 pacientes (4,5\%) (uma recidiva, dois casos com implantes no seio sagital superior pérvio, porção posterior). Complicações pós-operatórias foram observadas em 9 casos $(13,0 \%)$. Seis pacientes $(8,6 \%)$ apresentaram déficits motores transitórios; um paciente apresentou déficit permanente e síndrome cerebelar global; em um caso $(1,4 \%)$ ocorreu deiscência dos pontos cirúrgicos e infecção de ferida; e um outro caso $(1,4 \%)$ apresentou meningite bacteriana tratada com antibióticos endovenosamente. Óbito ocorreu em dois pacientes $(2,8 \%)$, um do sexo feminino e outro do sexo masculino, com idade de 75 e 70 anos e grau de edema I e II, respectivamente. Ambos tinham tumores parassagitais, falcinos.

A recorrência não foi avaliada devido ao curto intervalo de seguimento.

\section{DISCUSSÃO}

O termo meningioma foi usado inicialmente por H. Cushing em $1922^{6}$ para designar neoplasias originárias das meninges. A primeira descrição de um meningioma foi feita por Félix Paster em 


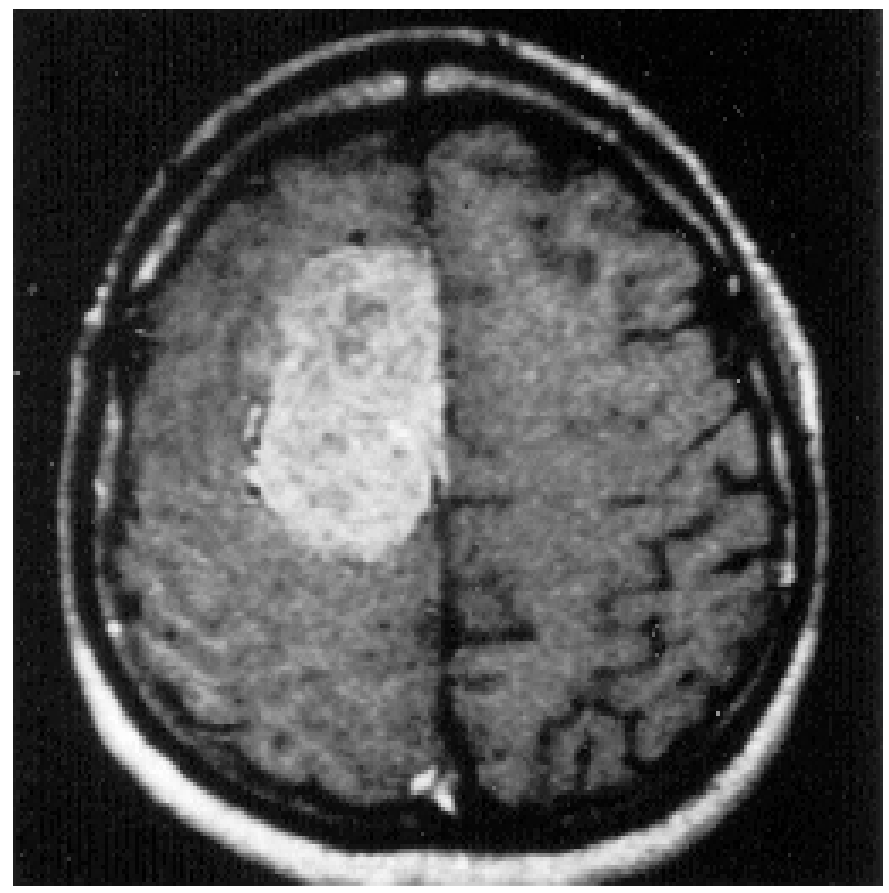

Fig 3. Corte axial de exame de ressonância magnética após injeção de gadolíneo evidenciando lesão tumoral com base de implantação na foice do cérebro. $O$ aspecto radiológico é característico de meningioma (ver texto).

$1614^{7}$. As primeiras tentativas de ressecção foram realizadas no século XVIII, incluindo aquela efetuada por Sir William MacEven ${ }^{8}$. Em 1887 W.W. Keen ressecou pela primeira vez um meningioma, com sucesso?.

Os meningiomas constituem tumores na maioria das vezes benignos e se originam de células da aracnóide, particularmente as das vilosidades. Têm sido sugeridas várias etiologias: traumática ${ }^{6,10,11}$, actínica $^{12-15}$, viral ${ }^{10,16-18}$ e genética (deleção envolvendo o cromossomo 22 ) $^{19}$.Uma vez que os meningiomas são mais comuns em mulheres ${ }^{1,20}$ e com frequência estão associados a carcinoma de mama ${ }^{21,22}$, hormônios sexuais também têm sido implicados em sua patogênese. Todavia, faltam evidências definitivas que esclareçam o verdadeiro papel de todos estes fatores na gênese dos meningiomas. Sua incidência é de 2:100000 ${ }^{23}$. Em grandes séries compreendem aproximadamente $20 \%$ de tumores intracranianos operados ${ }^{10}$. Acometem adultos de qualquer idade, embora raramente afetem também crianças ${ }^{24-27}$.

Apresenta-se clinicamente com convulsões, hemiparesia, perda visual, afasia e síndrome de hipertensão intracraniana. Há casos assintomáticos que podem se constituir em importantes dilemas terapêuticos.

O aspecto radiológico é bem descrito. À TC de crânio são tumores isointensos na fase não contrastada, multilobulados, adjacentes a estruturas durais onde se implantam. Realçam uniformemente à injeção do meio de contraste. Podem ser parcialmente calcificados e em até 15\% dos casos apresentam áreas de necrose ou hemorragia e cistos ${ }^{3}$. Margens mal definidas, invasão do parênquima e realce irregular quando presentes sugerem agressividade, embora os meningiomas malignos não possam ser distinguidos de outros subtipos através de exames de imagem ${ }^{28-30}$. À RNM 
são habitualmente hipo ou isointensos em T1; em T2 mostram-se iso ou hiperintensos. Após a injeção de contraste paramagnético, apresentam realce importante e usualmente homogêneo (Fig 3).

A TC e RNM mostram edema circunjacente em 46 a $92 \%$ das vezes ${ }^{31,32}$. Dentre os tumores que causam edema, o meningioma tem características únicas: é extra-axial, geralmente benigno e de crescimento lento. A magnitude do edema peritumoral produzido varia amplamente. Muitos fatores têm sido analisados como determinantes do edema, entre eles localização, tamanho do tumor, diferenciação histológica ${ }^{25,31,33}$ e vascularização pial ${ }^{34}$.

A natureza do edema é mais vasogênica que citotóxica, mostrando que a quebra de barreira hemato-encefálica pode representar o fator primário na gênese do edema ${ }^{35}$, mesmo se tratando de lesão extra-axial. Alguns autores postulam que o próprio tumor teria atividade secretória que seria responsável pelo desencadeamento de fenômenos vasogênicos ${ }^{36-38}$. Outros estudos tentam vincular a atividade de receptores de progesterona à patogênese do edema ${ }^{39,40}$. Não há estudos que mostrem significância estatística entre a ocorrência de edema e idade, sexo, agressividade, compressão vascular, tamanho ou localização do tumor ${ }^{37,38}$; no entanto, há aumento da tendência de ocorrência de edema em meningiomas de asa de esfenóide, convexidade (Fig 1), foice, região parassagital e região frontobasal ${ }^{30,31,41-43}$. Meningiomas de fossa posterior, suprasselares, e intraventriculares tendem a exibir menos edema ${ }^{30,31,44}$.

O tratamento dos meningiomas é cirúrgico e muitas vezes constitui grande desafio técnico, sendo o compromisso fundamental do cirurgião não acrescentar morbidade, já que são lesões muitas vezes benignas e completamente ressecáveis. A definição da abordagem, a extensão da ressecção e a dissecção da lesão de estruturas como tronco cerebral, seios venosos e nervos cranianos podem representar problemas técnicos consideráveis ${ }^{45}$. A ressecção completa usualmente é possível em tumores da convexidade, goteira olfatória e terço anterior do seio sagital. Tumores de tenda e fossa posterior também são susceptíveis de ressecção total; porém, a ressecção subtotal constitui por vezes o objetivo inicial em meningiomas de clivus, terço médio da asa do esfenóide e porção posterior do seio sagital superior ${ }^{18,46-48}$.

Embora em sua maioria sejam tumores benignos, os meningiomas podem ser neoplasias fatais. A mortalidade varia de $7-14 \%$ em diferentes séries ${ }^{10,49,50}$. A taxa de sobrevida é variável. Chan e Thompson ${ }^{51}$ relataram sobrevida média de 9 anos após a cirurgia. Simpson ${ }^{5}$ mostrou sobrevida pósoperatória média de $43 \%$ em 10 anos, com ressecção total. Mirimanoff e cols. ${ }^{52}$ obtiveram sobrevida de $77 \%$ em 10 anos, enquanto Kallio e cols. ${ }^{53}$ mostraram sobrevida de $63 \%$ em 15 anos.

A recorrência em meningiomas é comum e está diretamente relacionada ao grau de ressecção; mesmo após ressecção total a recorrência é estimada em até $15 \%{ }^{54}$. Se o tumor é completamente removido junto com a dura-máter e o osso acometido, a recorrência provavelmente se deve à presença de focos multicêntricos ${ }^{55}$. Simpson ${ }^{5}$ estabeleceu um sistema de gradação para ressecção de meningiomas que varia desde a sua completa remoção, juntamente com a dura-máter e o osso (Simpson I) até a simples biópsia da lesão (Simpson V) e obteve relação direta com a percentagem de recidiva (9\% em Simpson I a 40\% em Simpson IV). Outro fator determinante da recidiva é o tipo histológico. Acredita-se que os tipos angioblásticos e papilares apresentam tendência maior à recorrência ${ }^{3}$. Os meningiomas atípicos, assim como os meningiomas malignos e hemangiopericitomas, apresentam maior taxa de recidiva ${ }^{55}$.

Em nossa série foi obtida alta percentagem de ressecção completa - Simpson I e II em 95,5\% - provavelmente relacionada à elevada frequência relativa de meningiomas de convexidade nesta nossa casuística (40\%). Nos três casos $(4,5 \%)$ em que não foi possível a ressecção total, havia importante aderência do tumor ao seio sagital superior pérvio. Houve discreta predominância de casos com edema grau II $(38,7 \%)$ em nossa série. A média de idade em nosso estudo (58 anos) foi superior à encontrada na literatura $(45 \text { anos })^{55}$. As complicações $(n=9)$ observadas foram transitórias 
em sua maioria e não acrescentaram morbidade definitiva aos pacientes em oito casos. Um paciente de 75 anos apresentou isquemia cerebelar, distante do sítio operado. Apenas dois óbitos (4,2\%) ocorreram em nossa série, ambos casos de tumores falcinos em pacientes idosos (infarto venoso). Não houve correlação entre morbi-mortalidade e grau de edema em nosso estudo. Ojjeman ${ }^{48}$ descreveu 82 casos de meningiomas parassagitais, falcinos e de convexidade, obteve ressecção subtotal em 7 $(53,8 \%)$ de 13 meningiomas parassagitais operados, dois pacientes falecerem $(2,4 \%)$. Comparada com este estudo, nossa série mostrou maior taxa de ressecção total em meningiomas parassagitais $(89,7 \%$ versus $46,2 \%)$, embora a tentativa de ressecção total tenha resultado em maior taxa de mortalidade $(2,4 \%$ contra $2,8 \%)$.

Concluímos que a morbi-mortalidade em nossa série esteve relacionada à idade, a tumores falcinos e a tentativa de ressecção radical de tumores implantados em seio venoso pérvio. O grau de edema não foi determinante em aumentar as dificuldades técnicas ou elevar os índices de mortalidade.

\section{REFERÊNCIAS}

1. Kepes JJ. Meningiomas: biology, pathology and differential diagnosis. New York: Masson,1982.

2. Zimmerman HM. Brain tumors: their incidence and classification in man and their experimental production. Ann NY Acad Sci 1969;159:337-359.

3. Black PMcL. Meningiomas. Neurosurgery 1993;32:643-657.

4. Ide M, Jimbo M, Kubo O, Yamamoto M, Takeyama E, Imanaga H. Peritumoral brain edema and cortical damage by meningioma. Acta Neuropathol 1994;60(Suppl):369-372.

5. Simpson D. Recurrence of intracranial meninigiomas after surgical treatment. J Neurol Neurosurg Psychiatry 1957,20:22-39.

6. Cushing H, Eisenhardt L. Meninigiomas: their classification, regional behavior, life history and surgical end results. Sprigfield: Charles C. Thomas, 1938.

7. Netsky MG, Lapresele J. The first account of a meningioma. Bull Hist Med 1956;30:465-468.

8. Al-Rhodan NF, Laws ER Jr. The history of intracranial meningiomas. In Al-Mefty O (ed). Meningiomas. Nova York: Raven Press, 1991:1-7.

9. Bingham WF. WW Keen and the dawn of American neurosurgery. J Neurosurg 1986;64:705-712.

10. Rachlin JR, Roseblum ML. Etiology and biology of meningiomas. In Al-Mefty O (ed). Meningiomas. Nova York: Raven Press, 1991:22-37.

11. Schiffer J, Avidan D, Rapp A. Posttraumatic meningioma. Neurosurgery 1985,17:84-87.

12. Modan B, Baidatz D, Mart H, Steinitz R, Levin SG. Radiation induced head and neck tumors. Lancet 1974;1:277-279.

13. Ojeda VJ, Stokes BA, Woodland P. Meningioma occurring twenty-three years after a successfully treated cerrebellar medulloblastoma: case report. Aust N Z J Surg 1987;57:133-135.

14. Partington MD, Davis DH. Radiation-induced meningioma after treatment for pituitary adenoma: case report and literature review. Neurosurgery 1990;26:329-331.

15. Sridhar K, Ramamurthi B. Intracranial meningioma subsequent to radiation for a pituitary tumor: case report. Neurosurgery 1989;25:643-645.

16. Barbanti-Brodano G, Silini E, Mottes M. Probes to evaluate the possible association of papovavirus with human tumors: In Gallo RC (ed). Monoclonal and DNA probes in diagnostic and preventive medicine. New York: Raven Press 1987:147-155.

17. Ibelgaufts H, Jones KW, Maitland N, Shaw JF. Adenovirus related RNA sequences in human neurogenic tumors. Acta Neuropathol (Berl) 1982;56:113-117.

18. Maxwell RE, Chou SN. Preoperative evaluation and management of meningiomas. In Schmidek HH, Sweet WH (eds). Operative neurosurgical techniches. New York: Grune \& Stratton 1988:547-554.

19. Collins VP, Mordenskjold M, Dumanski JP. The molecular genetics of meninigiomas. Brain Pathology 1990;1:9-24.

20. Russel DS, Rubenstein LF. Pathology of tumors of the nervous system. Baltimore. Willians \& Wilkins, 1982.

21. Rubibstein $\mathrm{AB}$, Schein M, Reichenthal E. The association of carcinoma of the breast with meninigioma. Surg Gynecol Obstet 1989;169:334-336.

22. Schoenberg BS, Christine BW, Whishant JP. Nervous system neoplasms and primary malignancy of other sites: the unique association between meningiomas and breast cancer. Neurology 1975;705-712

23. Rohringer M, Sutherland GR, Louw DF, Sima AF. Incidence and clinicopathological features of meningiomas. J Neurosurg 1989;71:665-672.

24. Doty JR, Schut L, Brice DA, Sutton LN. Intracranial menigiomas of childhood and adolescence. Progr Exp Tumor Res 1987;30:247-254.

25. Drake JM, Hendrick EB, Becker LE, Chuang SH, Hoffman JH, Humpheys RP. Intracranial meningiomas in children. Pediatr Neurosci 1985;12:134-139.

26. Ferrante L, Acqui M, Artico M, Mastronardi L, Fortuna A. Pediatric intracranial meningiomas. Br J Neurosurg 1989;3:189-196.

27. Ferrante L, Acqui M, Artico M, Mastronardi L, Rochii G, Fortuna A. Cerebral meningiomas in children. Childs Nerv Syst 1989;5:83-86.

28. Nakasu S, Hirano A, Llena JF, Shimura T, Handa J. Interface between the meningioma and the brain. Surg Neurol 1989;32:206-212. 
29. Salcman M. Malignant meningiomas. In Al-Mefty O (ed). Meningiomas. New York: Raven Press 1991:75-85.

30. Maiuri F, Gangemi M, Cirillo S, et al. Cerebral edema associated with meningiomas. Surg Neurol 1987;27:64-68.

31. Bradac Gb, Ferszt R, Bender A, Schörner W. Peritumoral edema in meningiomas: a radiological and histological study. Neuroradiology 1986;28:304-312.

32. Vakili ST, Muller J. Intracytoplasmic lumina in meningioma: an ultrastructural and immunohistological study. Neurosurgery 1988;23:180-184.

33. Inamura T, Nishio S, Takeshita I, Fujiwara S, Fukui M. Peritumoral brain edema in meningiomas: influence of vascular supply on its development. Neurosurgery 1992;31:179-185.

34. Bitzer M, Wöckel L, Luft AR, et al. The importance of pial blood supply to the development of peritumoral brain edema in menigiomas. J Neurosurg 1997;87:368-373.

35. Hossman KA, Welcher W, Wilmes F. Experimental peritumorous edema: morphological and pathophysiological observations. Acta Neuropathol 1979;45:195-203.

36. Philipon J, Foncin JF, Grob R, Sraw A, Pertuiset BF. Cerebral edema associated with meningiomas: possible role of a secretory-excretory phenomenon. Neurosurgery 1984;14:295-301.

37. King WA, Black KL. Peritumoral edema with meningiomas. In Schimidek H (ed). Meningiomas and their surgical treatment. Philadelphia: WB Saunders 1991;55-63.

38. Lindley JG, Challa VR, Kelly DL. Meningiomas and brain edema. In Al-Mefty O (ed). Meningiomas. New York: Raven Press 1991:59-73.

39. Benzel EC, Gelder FB. Correlation between sex hormones binding and peritumoral edema in intracranial meningiomas. Neurosurgery 1988;23:169-174.

40. Jaaskelainen J, Laasonen E, Karkkainen J, Haltia M, Troup H. Hormone treatment of meningiomas: lack of response to medroxyprogesterone acetate. A pilot study of five cases. Acta Neurochir (Wien) 1986;80:35-41

41. Fine M, Brazis P, Palacios E, Neri G. Computed tomography of sphenoid wing meningiomas: tumor location related to distal edema. Surg Neurol 1980;13:385-390.

42. Gilbert JJ, Paulseth JE, Coates RK, Malott D. Cerebral edema associated with meningiomas. Neurosurgery 1983;12:599-605.

43. Stevens JM, Ruiz JS, Kendall BE. Observations on peritumoral oedema in meningioma: Part II. Mechanisms of oedema production. Neuroradiology 1983;25:125-131.

44. Vassilouthis J, Ambrose J. Computerized tomography scanning appearances of intracranial meningiomas: an attempt to predict the histological features. J Neurosurg 1979;50:320-327.

45. Ojjeman RG, Black PM. Difficult decisions in managing patients with benign brain tumors. In Black PM (ed). Clinical neurosurgery. Baltimore: Willians \& Wilkins 1989; 35:254-284.

46. MacCarty CS, Taylor WF: Intracranial meningiomas: experiences at the Mayo Clinic. Neurol Med Chir 1979;19:569-574.

47. Maxwell RE, Chou SN. Convexity meningiomas and general principles of meningioma surgery. In Schimidek HH, Sweet WH (eds). Operative neurosurgical techniques. New York: Grune \& Stratton 1988:555-562.

48. Ojjeman RG. Meninigiomas: clinical features and surgical management. In Wilkins RH, Rengachary SS (eds). Neurosurgery. New York: McGraw-Hill 1985:635-654.

49. Jan M, Bazeze V, Saudeau D, Autret A, Bertrand P, Gouaze A. Outcome of intracranial meningiomas in adults: retrospective study of a medicosurgical series of 161 meningiomas. Neurochirurgie 1986;32:129-134.

50. Pertuiset B, Farah S, Clayes L, Goutorbe J, Metzger J, Kujas M. Operatibility of intracranial meningiomas: personal series of 353 cases. Acta Neurochir (Wien) 1985;76:2-11.

51. Chan RC, Thompson GB. Morbidity, mortality and quality of life following surgery for intracranial meningiomas: a restrospective study in 257 cases. J Neurosurg 1984;60:52-60.

52. Mirimanoff RO, Dosoretz DE, Linggood RM, Ojjeman R, Martuza R. Meningioma: analysis of recurrence and progression following neurosurgical resection. J Neurosurg 1985;62:18-24.

53. Kallio M, Sankila R, Hankulinen T, Jaaskelainen J. Factors affecting operative and excess long-term mortality in 935 patients with intracranial meninigioma. Neurosurgery 1992;31:2-12.

54. Yamashita J, Handa H, Iwaki K, Abe M. Recurrence of intracranial meningiomas, with special reference to radiotherapy. Surg Neurol 1980;14:33-40.

55. Al-Mefty O, Origatano TC. Meningiomas. In Rengachary SS e Wilkins RH (eds). Principles of neurosurgery, London. Wolfe 1994;28:1-14. 